Description A 29-year-old, nulliparous woman with stage IB1 cervical cancer received the LRT plus lymphadenectomy with enclosed colpotomy and without manipulator. Instead of using a uterine manipulator, uterine suspension was adopted. We suture bilateral round ligaments to form a coil with the anterior wall of the uterus which help remain the integrity of the uterus. After the branches of the uterine artery were separated, the uterine branch was retained.Reconstruction of the residual cervix and vagina was performed by the 'cuff-sleeve' suture method. The anterior and posterior walls of the vagina were sutured with 2 ' $U$ ' shapes respectively with special care given to the uterine branches of the uterine artery. At the end of the surgery, a stent was set trans-vaginally in case of cervical stenosis. The 'neocervix' was restored to its original anatomy after 6 months.Till now, reproductive function and sexual health is satisfied for the patient.

Conclusions LRT with unique modifications is safe and feasible for patients with early stage cervical cancer to preserve fertility. Further study shall be in place to better evaluate the postoperative efficacy and pregnancy outcomes of such procedure.

\section{SF002/\#332 SENTINEL LYMPH NODE MAPPING FOR CERVICAL CANCER: A PRACTICAL ILLUSTRATION OF USING ICG DURING A LAPAROTOMY}

${ }^{1} \mathrm{M}$ Burling, ${ }^{2} \mathrm{D}$ Krishnan*, 'L Foster, ${ }^{1} \mathrm{~A}$ Brand, ${ }^{2} \mathrm{U}$ Herbst. 'Westmead Hospital, Gynaecological Oncology, Westmead, Australia; 'Westmead Hospital, Gynaeoncology, Westmead, Australia

10.1136/ijgc-2021-IGCS.46

We have created a surgical video to demonstrate the sentinel lymph node mapping in cervical cancer during a laprotomy. Sentinel lymph node (SLN) mapping is an emerging technique to be used in the surgical management of women with cervical cancer. Multiple studies including SENTICOL 2 has shows the accuracy of using SLN biopsy in cervical cancer treatment [1]. This approach has mainly been used with minimally invasive surgery. However, since the publication of the LACC trial in 2018, there has been a evidence based shift to open radical hysterectomy for early stage cervical cancer [2]. We aim to display how to perform SLN biopsy with indocyanine green (ICG) dye, using a laparoscopic camera even in open surgery. The video footahe were gleaned from an unedited surgical film recorded at our institute. We conclude that SLN mapping and biopsy with ICG during a laparotomy is feasible and achievable.

\section{SF003/\#661 TOTAL PELVIC EXENTERATION AND PERINEAL RECONSTRUCTION FOR RECURRENT CERVICAL CANCER}

A Thomas*, R Chandy, A Sebastian, R Mittal, JK Ramireddy, R Paul Mukha, M Lamba, A Peedicayil. Christian Medical College, Vellore, Gynecologic Oncology, Vellore, India

\subsection{6/ijgc-2021-IGCS.47}

Background and Introduction Salvage procedures for recurrent and residual cervical cancer following radical chemo radiation can be offered to patients with motivation and willingness to undergo massive operative procedures. It needs the skill of a multidisciplinary team who can contribute to various aspects of this complicated procedure. Description of the technique Here we describe Total Pelvic exenteration in a 49 year old lady with residual disease of cervical cancer who after chemo radiation for a bulky stage IIIB $(8 \mathrm{~cm})$ endocervical adenocarcinoma of the cervix was found to have central residual disease infiltrating bladder, vagina, anal canal and rectum. She was offered total exenteration after 3 cycles of chemotherapy with Paclitaxel and Carboplatinum after ruling out metastatic disease with a PET-CT. She was operated upon by a team comprising of urologist, colorectal surgeon, gynaecologic oncologists and plastic surgeon. She underwent Total Pelvic exenteration with enbloc removal of uterus with vagina, urinary bladder, urethra, rectum and and anal canal. The ureters were diverted into an ileal conduit by Wallace technique and the colon was diverted into a Hartmans Pouch The vulval defect was reconstructed by gracilis myocutanaeous flap repair over a vicryl mesh. Post operatively her wound healed well and she was discharged on $8^{\text {th }}$ post-operative day. Surgical margins were free of tumour on histopathology. She is alive and well on follow up after 30 months.

Conclusions Salvage procedures for residual and recurrent cervical cancers can result in good survival and quality of life in motivated and surgically fit patients.

\section{SF004/\#153 THE USE OF LIGHTED URETERAL CATHETERS IN DIFFICULT MINIMALLY INVASIVE GYNECOLOGIC ONCOLOGIC SURGERY}

${ }^{1} \mathrm{~J}$ Mejia-Gomez ${ }^{*},{ }^{2} \mathrm{G}$ Lennox, ${ }^{2} \mathrm{~K}$ Pulman, ${ }^{2} \mathrm{~S}$ Sinasac, ${ }^{2} \mathrm{~T}$ Feigenberg. ${ }^{1}$ Mount Sinai Hospital/ University of Toronto, Obstetrics and Gynecology, Toronto, Canada; ${ }^{2}$ Trillium Health Partners/University of Toronto, Gynecologic Oncology, Mississauga, Canada

\subsection{6/ijgc-2021-IGCS.48}

Introduction Over the past decade, the utility of minimally invasive surgery (MIS), laparoscopy and robotics, has significantly increased in gynecologic oncology surgeries. MIS has many advantages Over open surgery including a significant reduction in the length of hospital stay, fewer post-operative complications, and increased patient satisfaction. Ureteral injuries are amongst the most common complication of gynecologic procedures. The presence of large pelvic masses, significant obesity, adhesions and previous radiation can increase the risk of ureteral injuries during gynecologic oncology procedures. Description Our objective is to demonstrate the technique of stent insertion and use. In addition we will demonstrate the use of lighted ureteral stents in difficult selective minimally invasive gynecologic oncology procedures such as surgery on morbidly obese patients, very large pelvic masses, management of adhesions and surgery for patients who were previously treated with pelvic radiation. We will demonstrate the use of lighted ureteral catheters in both laparoscopic and robotic procedures.

Conclusion Lighted Urethral stents can assist the surgeon in identifying the ureter, reduce the needs for extensive retroperitoneal dissection and potentially reduce rates of ureteral injuries. The usage of lighted ureteral catheters/stents are supported in both standard laparoscopic and robotic surgery. Techniques that can support the surgeon to accurately identify the ureters are paramount. 